\title{
EXISTENCE RESULTS FOR DIFFERENTIAL EQUATIONS WITH REFLECTION OF THE ARGUMENT
}

\author{
DONAL O'REGAN
}

(Received 31 January 1992)

Communicated by P. E. Kloeden

\begin{abstract}
Existence principles are given for systems of differential equations with reflection of the argument. These are derived using fixed point analysis, specifically the Nonlinear Alternative. Then existence results are deduced for certain classes of first and second order equations with reflection of the argument.
\end{abstract}

1991 Mathematics subject classification (Amer. Math. Soc.): 34A12, 34B15, 34K05, 34K10.

\section{Introduction and Preliminaries}

This paper establishes some general existence principles for systems of differential equations with reflection of the argument. Then applications are given to certain classes of first and second order equations with reflection of the argument. For a detailed account on the above subject see $[1,2,11,12]$ and their references. The problems we consider have the form

$$
\left\{\begin{array}{l}
y^{(k)}(t)=f\left(t, y(t), y(-t), \cdots, y^{(k-1)}(t), y^{(k-1)}(-t)\right), \quad-T \leq t \leq T \\
y \in \mathscr{B}
\end{array}\right.
$$

where $\mathscr{B}$ specifies certain initial and/or boundary conditions and where $f$ is either continuous or a Carathéodory function.

For this paper $R^{n}$ denotes Euclidean $n$-space and $|x|$ denotes the Euclidean norm, $C^{m}=C^{m}\left([-T, T], R^{n}\right)$, where the range space of the functions will be clear from the context, is the Banach space of functions $u$ such that $u^{(m)}$ is continuous on $[-T, T]$ with norm

$$
|u|_{m}=\max \left\{|u|_{0}, \cdots,\left|u^{(m)}\right|_{0}\right\}
$$

(C) 1994 Australian Mathematical Society 0263-6115/94 \$A2.00+0.00 
where $|u|_{0}=\max \{|u(t)|:-T \leq t \leq T\}$. We also define $C_{0}^{m}=\left\{u \in C^{m}: u(0)=0\right\}$ and of course as usual $C=C^{0}$ and $C_{0}^{0}=C_{0}$. For $1 \leq p<\infty, L^{p}[-T, T]$ is the Banach space of functions $u$ such that $|u|^{p}$ is Lebesgue measurable on $[-T, T]$ with norm

$$
\|u\|_{p}=\left\{\int_{-T}^{T}|u(x)|^{p} d x\right\}^{\frac{1}{p}}
$$

and $L^{\infty}[-T, T]$ is the Banach space of essentially bounded functions $u$ with $\|u\|_{\infty}$ the usual essential supremum norm. For simplicity when $p=2$, we write $\|u\|=$ $\|u\|_{2}$. Finally $W^{k, p}[-T, T]$ is the Sobolev class of functions $u$ with $u^{(k-1)}$ absolutely continuous and $u^{(k)} \in L^{p}[-T, T]$.

We will use the following version of the change-of-variable theorem; see [5] for proof.

LEMMA 0.1. Let $g:[a, b] \rightarrow[A, B]$ and $h:[A, B] \rightarrow R$, where $g$ is absolutely continuous, $h$ is measurable and $(h \circ g) g^{\prime}$ is Lebesgue measurable on $[a, b]$. Then $h$ is integrable on the interval with end points $g(a)$ and $g(b)$ and

$$
\int_{g(a)}^{g(b)} h(u) d u=\int_{a}^{b} h(g(t)) g^{\prime}(t) d t .
$$

Now in $(0.1)$ we assume the nonlinear term $f$ is either a continuous function or a Carathéodory function.

DEFINITION. Let $m$ be a positive integer and $p \in[1, \infty]$. A function $g:[-T, T] \times$ $R^{m n} \rightarrow R^{n}$ is an $L^{p}$-Carathéodory function (or a Carathéodory function for short) provided:

if $g=g(t, z)$, then

(a) the map $z \mapsto g(t, z)$ is continuous for almost all $t \in[-T, T]$;

(b) the map $t \mapsto g(t, z)$ is measurable for all $z \in R^{m n}$;

(c) for each $r>0$ there exists $h_{r} \in L^{p}[-T, T]$ such that $|z| \leq r$ implies that $|g(t, z)| \leq h_{r}(t)$ for almost all $t \in[-T, T]$.

Each Carathéodory function $g:[-T, T] \times R^{(2 k) n} \rightarrow R^{n}$ induces an operator (called the Carathéodory operator associated with $g$ ) $N_{g}: C^{k-1} \rightarrow C_{0}$ defined by

$$
\left(N_{g} y\right)(x)=\int_{0}^{x} g\left(s, y(s), y(-s), \cdots, y^{(k-1)}(s), y^{(k-1)}(-s)\right) d s .
$$

The condition (a) and (b) in the above definition imply that $g\left(s, y_{1}(s), y_{2}(s), \cdots\right.$, $\left.y_{2 k}(s)\right)$ is measurable for any measurable functions $y_{1}(s), \cdots, y_{2 k}(s)$. Thus if $y \in$ $C^{k-1}\left([-T, T], R^{n}\right)$, then (c) implies that the integrand is integrable on $[-T, T]$ and consequently $N_{g}: C^{k-1} \rightarrow C_{0}$ as asserted. Essentially the same reasoning as in [7] or $[9,10]$ immediately yields: 
THEOREM 0.2. Let $g:[-T, T] \times R^{(2 k) n} \rightarrow R^{n}$ be a Carathéodory function and $N_{g}: C^{k-1} \rightarrow C_{0}$ its associated operator. Then $N_{g}$ is continuous and completely continuous.

If $f$ is continuous, by a solution to $(0.1)$ we mean a classical solution $y$ with a continuous $k^{\text {th }}$ derivative, while if $f$ is a Carathéodory function, a solution will mean a function $y$ which has an absolutely continuous $(k-1)^{s t}$ derivative such that $y$ satisfies the differential equation almost everywhere in $[-T, T]$. Of course $y$ must also satisfy the stated boundary conditions. If $f$ is $L^{p}$-Carathéodory then any solution to $(0.1)$ will automatically have its $k^{t h}$ derivative in $L^{p}$ and hence be an element of $W^{k, p}[-T, T]$.

In $(0.1)$ we treat initial and/or boundary conditions $\mathscr{B}$ of the following type. For each $i=1, \cdots, k$ let $U_{i}: C^{k-1}\left([-T, T] ; R^{n}\right) \rightarrow R^{n}$ be a continuous linear form such that $U_{i}(\theta(t) v)=U_{i}(\theta(t)) v$ for each $k-1$ times differentiable, real valued function $\theta$ and for each vector $v$ in $R^{n}$. We say a function $u \in C^{m}[-T, T]$ for $m \geq k-1$ satisfies the boundary conditions $\mathscr{B}$ and write $u \in \mathscr{B}$ if $U_{i}(u)=\gamma_{i}$ for $i=1, \cdots, k$ where $\gamma_{i}$ are given constants. The corresponding homogeneous boundary conditions where each $\gamma_{i}=0$ are denoted by $\mathscr{B}_{0}$. Thus, $u \in \mathscr{B}_{0}$ if $U_{i}(u)=0$ for $i=1, \cdots, k$. Finally, given a space $\mathscr{F}$ of suitably differentiable functions, we denote by $\mathscr{F}_{\mathscr{B}}$ the subset of those functions in $\mathscr{F}$ which satisfy the boundary conditions $\mathscr{B}$.

Our analysis of (0.1) involves two related operators $\Lambda$ and $L$ defined as follows:

$$
\begin{array}{ll}
\Lambda: C_{\mathscr{B}_{0}}^{k} \rightarrow C, & \Lambda y=y^{(k)} \\
L: C_{\mathscr{B}}^{(k-1)} \rightarrow C_{0}, & (L y)(t)=y^{(k-1)}(t)-y^{(k-1)}(0)
\end{array}
$$

where $\mathscr{B}$ denotes the boundary conditions $U_{i}(y)=\gamma_{i}$ for $i=1, \cdots, k$. Notice that $\Lambda$ is a linear operator while $L$ is affine.

LEMMA 0.3. Let $\left\{z_{j}(x)\right\}_{j=1}^{k}$ be a basis of solutions for the scalar equation $z^{(k)}=0$. Then $\Lambda: C_{\mathscr{B}_{0}}^{k} \rightarrow C$ is invertible if and only if $\operatorname{det}\left[U_{i}\left(z_{j}\right)\right] \neq 0$.

PROOF. The equation $\Lambda y=0$ holds precisely when $y=\sum_{j=1}^{k} z_{j}(x) a_{j}$ where $a_{j} \in R^{n}$ satisfy

$$
0=U_{i}\left[\sum_{j=1}^{k} z_{j}(x) a_{j}\right]=\sum_{j=1}^{k} U_{i}\left(z_{j}(x)\right) a_{j}
$$

for each $i=1, \cdots, k$, and the result follows.

LEMMA 0.4. If $\Lambda: C_{\mathscr{B}_{0}}^{k} \rightarrow C$ is invertible then so is $L: C_{\mathscr{B}}^{k-1} \rightarrow C_{0}$.

Proof. The equations $y^{(k-1)}(x)-y^{(k-1)}(0)=g(x)$ with $g \in C_{0}$ have general solution $y(x)=\sum_{j=1}^{k} z_{j}(x) a_{j}+G(x)$. Where of course $G(x)=g(x)$ when $k=1$, 
$G(x)=\int_{0}^{x} g\left(s_{1}\right) d s_{1}$ when $k=2$ and $G(x)=\int_{0}^{x} \int_{0}^{s_{k-1}} \cdots \int_{0}^{s_{2}} g\left(s_{1}\right) d s_{1} d s_{2} \cdots d s_{k-1}$ when $k \geq 3$, and $z_{j}(x)$ are as in Lemma 0.3 . The function $y(x)$ satisfies the boundary conditions $\mathscr{B}$ if and only if $U_{i}(y)=\gamma_{i}$ for $i=1,2, \cdots, k$; that is $\sum_{j=1}^{k} U_{i}\left(z_{j}(x)\right) a_{j}=$ $\gamma_{i}-U_{i}(G(x)), i=1, \cdots, k$. In view of Lemma 0.3 there are unique choices for the vectors $a_{j} \in R^{n}$ such that $y(x)$ solves $L y=g$. Therefore given $g \in C_{0}$ there always exists $y \in C_{\mathscr{T}}^{k-1}$ such that $L y=g$. The difference of two such solutions, say $z$, clearly belongs to $C_{\mathscr{B}_{0}}^{k}$ and satisfies $\Lambda z=0$. Thus $z=0$ and we conclude that $L$ is both one-to-one and onto and hence invertible.

The general existence principles for differential equations with reflection of the argument are derived using topological methods, specifically the Nonlinear Alternative $[4,9,10,14]$. We note first, however, that by a map being compact we mean it is continuous with relatively compact range. Let $K$ denote a convex subset of a normed, linear space $E . U$ is an open subset of $K$ and $\bar{U}$ and $\partial U$ are the closure of $U$ in $K$ and the boundary of $U$ in $K$.

THEOREM 0.5. (Nonlinear Alternative) Let $N: \bar{U} \rightarrow K$ be a compact map, $p \in U$, and $N_{\lambda}(u)=N(u, \lambda): \bar{U} \times[0,1] \rightarrow K$ a family of compact maps with $N_{1}=N$ and $N_{0}(u)=p$, the constant map to $p$. Then either

(i) $N$ has a fixed point in $\bar{U}$; or

(ii) there is a point $u \in \partial U$ and $\lambda \in(0,1)$ such that $u=N_{\lambda} u$.

Now associated with the system $(0.1)$ we have a family of problems $(0.1)_{\lambda} \quad\left\{\begin{array}{l}y^{(k)}(t)=\lambda f\left(t, y(t), y(-t), \cdots, y^{(k-1)}(t), y^{(k-1)}(-t)\right), \quad-T \leq t \leq T \\ y \in \mathscr{B}\end{array}\right.$ where $\lambda \in[0,1]$. Evidently, $y$ is a solution to $(0.1)_{\lambda}$ if and only if it satisfies the equation

$$
y^{(k-1)}(t)-y^{(k-1)}(0)=\lambda \int_{0}^{t} f\left(s, y(s), y(-s), \cdots, y^{(k-1)}(s), y^{(k-1)}(-s)\right) d s
$$

and $y \in C_{\mathscr{B}}^{k-1}$. Assume that $\Lambda: C_{\mathscr{B}_{0}}^{k} \rightarrow C$ is invertible so Lemma 0.4 leads immediately to the equivalence

$(0.1)_{\lambda}$ is equivalent to $L y=\lambda N_{f} y$ is equivalent to $y=N_{\lambda} y$

where $N_{\lambda}=L^{-1} \lambda N_{f}: C^{k-1} \rightarrow C^{k-1}$ is continuous and completely continuous by Theorem 0.2. Now, an application of the Nonlinear Alternative yields:

THEOREM 0.6. Let $f:[-T, T] \times R^{(2 k) n} \rightarrow R$ be continuous or a Carathéodory function. Assume furthermore that $\Lambda: C_{\mathscr{B}_{0}}^{k} \rightarrow C$ is invertible and that $h$ is the unique solution to $L h=0$. Then each of the following three statements is valid. 
(A) Let $U \subset C_{\mathscr{B}}^{k-1}$ be a bounded, open set with $h \in U$. Then either

(i) (0.1) has a solution $y$ in $\bar{U}$; or

(ii) there exists $\lambda \in(0,1)$ and a solution $y$ to $(0.1)_{\lambda}$ such that $y \in \partial U$.

(B) Let $\Omega$ be a bounded open set in $R^{(2 k) n}$ with $\left(h(x), h(-x), \cdots, h^{(k-1)}(x), h^{(k-1)}(-x)\right)$ $\in \Omega$ for all $x$ in $[-T, T]$. Then either

(i) (0.1) has a solution $y$ with $\left(y(x), y(-x), \cdots, y^{(k-1)}(x), y^{(k-1)}(-x)\right) \in \bar{\Omega}$ for $x \in[-T, T]$; or

(ii) there exists $\lambda \in(0,1)$ and a solution $y$ to $(0.1){ }_{\lambda}$ such that $(y(x), y(-x)$, $\left.\cdots, y^{(k-1)}(x), y^{(k-1)}(-x)\right) \in \bar{\Omega}$ for all $x$ in $[-T, T]$ and for some $x_{0}$ in $[-T, T],\left(y\left(x_{0}\right), y\left(-x_{0}\right), \cdots, y^{(k-1)}\left(-x_{0}\right)\right) \in \partial \Omega$.

(C) Suppose there is a constant $M$ such that for every $\lambda \in(0,1)$, any solution $y$ to $(0.1)_{\lambda}$ we have $|y|_{k-1}<M$. Then $(0.1)$ has a solution $y$ such that $|y|_{k-1} \leq$ $\max \left\{M,|h|_{k-1}\right\}$.

ProOF. To prove (A), let $E=C^{k-1}$ and $K=C_{\mathscr{B}}^{k-1}$ which is a convex subset of $E$. Let $N: L^{-1} N_{f}: \bar{U} \rightarrow K$ and $N_{\lambda} u=N(u, \lambda)=L^{-1} \lambda N_{f} u$ define maps from $\bar{U} \times[0,1] \rightarrow K$. Since $U$ is bounded the comments following $(0.2)$ show that all these maps are compact. Also we have $N_{0} u=L^{-1} 0=h$, the constant map to $h \in U$. Thus Theorem 0.5 applies to these compact maps, but by (0.2), alternative (i) and (ii) in Theorem 0.5 are precisely alternative (i) and (ii) in (A) above. This establishes (A).

To verify (B), let $U=\left\{y \in C_{\mathscr{B}}^{k-1}:\left(y(x), y(-x), \cdots, y^{(k-1)}(x), y^{(k-1)}(-x)\right) \in \Omega\right.$ for $x \in(-T, T)\}$. Apply (A) with this $U$ to obtain (B).

Finally, apply (B) with $\Omega=\left\{w=\left(w_{1}, \cdots, w_{2 k}\right) \in R^{(2 k) n}:\left|w_{i}\right|<\max \left\{M,|h|_{k-1}\right\}\right\}$ and observe that that choice of $\Omega$ excludes (ii) in (B) to confirm (C).

The next few sections develop existence results for interesting classes of first and second order differential equations with reflection of the argument or systems of such equations.

\section{Initial Value Problems}

Consider a first order differential system with reflection of the argument of the form

$$
\left\{\begin{array}{l}
y^{\prime}(t)=\eta(t) f(t, y(t), y(-t)), \quad-T \leq t \leq T \\
y(0)=r
\end{array}\right.
$$

where $r \in R^{n}$. 
THEOREM 1.1. Let $1 / p+1 / q=1, f:[-T, T] \times R^{2 n} \rightarrow R^{n}$ be an $L^{p_{-}}$ Carathéodory function, $\eta \in L^{q}[-T, T]$ with $\eta \geq 0$. Assume $\psi:[0, \infty) \rightarrow(0, \infty)$ is a nondecreasing Borel function such that

$$
\left|f\left(s, u_{1}, u_{2}\right)\right| \leq \psi\left(\left|u_{1}\right|+\left|u_{2}\right|\right)
$$

for almost all $s$ in $[-T, T]$ and all $u_{1}, u_{2} \in R^{n}$. Then (1.1) has a solution y defined on $[-T, T]$ provided $T$ satisfies

$$
\int_{0}^{T}[\eta(s)+\eta(-s)] d s<\int_{2|r|}^{\infty} \frac{d u}{\psi(u)} .
$$

PROOF. First recall that by a solution to (1.1) we mean a function $y \in C_{\mathscr{B}}([-T, T]$, $R^{n}$ ) which is absolutely continuous on $[-T, T]$ and satisfies the differential equation almost everywhere on $[-T, T]$. Theorem $0.6(C)$ implies that a solution to (1.1) will exist if we can establish an a priori bound independent of $\lambda \in(0,1)$ for any solution to the family of problems

$$
\left\{\begin{array}{l}
y^{\prime}(t)=\lambda \eta(t) f(t, y(t), y(-t)), \quad-T \leq t \leq T \\
y(0)=r .
\end{array}\right.
$$

Note that Theorem 0.6 applies because $\eta f$ is an $L^{1}$-Carathéodory function by Hölders inequality and for $\mathscr{B}$ we may take the initial condition $U_{1}(u)=u(0)=r$. Then $\Lambda: C_{\mathscr{B}_{0}}^{1} \rightarrow C$ given by $\Lambda y=y^{\prime}$ is clearly invertible. In view of Theorem $0.6(\mathrm{C})$ we need an a priori bound for solutions to $(1.1)_{\lambda}$ in the $C[-T, T]$ norm. Let $y(t)$ be a solution to $(1.1)_{\lambda}$. Then for almost all $t \in[-T, T]$

$$
\left|y^{\prime}(t)\right| \leq \eta(t) \psi(|y(t)|+|y(-t)|) \quad \text { and } \quad\left|y^{\prime}(-t)\right| \leq \eta(-t) \psi(|y(-t)|+|y(t)|) .
$$

Consequently, for almost all $t \in[-T, T]$

$$
\left|y^{\prime}(t)\right|+\left|y^{\prime}(-t)\right| \leq[\eta(t)+\eta(-t)] \psi(|y(t)|+|y(-t)|) .
$$

Let $t \in(0, T]$ so $-t \in[-T, 0)$ and so $y(t)=y(0)+\int_{0}^{t} y^{\prime}(s) d s$ with $y(-t)=$ $y(0)+\int_{0}^{-t} y^{\prime}(s) d s$ yields

$$
|y(t)| \leq|r|+\int_{0}^{t}\left|y^{\prime}(s)\right| d s \quad \text { and } \quad|y(-t)| \leq|r|+\int_{-t}^{0}\left|y^{\prime}(s)\right| d s .
$$

Combining (1.2), (1.3) and the fact that $\psi$ is a nondecreasing function yields for almost all $t \in[0, T]$

$$
\frac{\left|y^{\prime}(t)\right|+\left|y^{\prime}(-t)\right|}{\psi\left(2|r|+\int_{-t}^{t}\left|y^{\prime}(s)\right| d s\right)} \leq \eta(t)+\eta(-t) .
$$


Integration from 0 to $T$ and use of Lemma 0.1 gives with $\rho(t)=2|r|+\int_{-t}^{t}\left|y^{\prime}(s)\right| d s$,

$$
\int_{2|r|}^{\rho(t)} \frac{d u}{\psi(u)} \leq \int_{0}^{T}[\eta(t)+\eta(-t)] d t<\int_{2|r|}^{\infty} \frac{d u}{\psi(u)}
$$

and we conclude that there is a constant $M$ independent of $\lambda \in(0,1)$ such that $\rho(t) \leq M$ for $0 \leq t \leq T$. Consequently for $t \in[0, T],(1.3)$ implies

$$
|y(t)| \leq \rho(t) \leq M \quad \text { and } \quad|y(-t)| \leq \rho(t) \leq M
$$

and so $|y(t)| \leq M$ for $t \in[-T, T]$ that is the $C[-T, T]$ norm of $y$ is bounded by $M$. Hence (1.1) has a solution on $[-T, T]$.

COROLlARY 1.2. Assume $\eta \in L^{1}[-T, T]$ with $\eta \geq 0$ and continuous on $(-T, T)$, that $f$ is continuous and the growth rate restriction of Theorem 1.1 holds. Then (1.1) has a solution $y \in C[-T, T] \cap C^{1}(-T, T)$. Moreover, this result is best possible for the class of initial value problems with reflection of the argument satisfying the growth condition $\left|f\left(t, u_{1}, u_{2}\right)\right| \leq \psi\left(\left|u_{1}\right|+\left|u_{2}\right|\right)$, with $\psi$ continuous.

PROOF. By Theorem 1.1 there is a solution $y(t)$ which is absolutely continuous on $[-T, T]$ and satisfies the differential equation in (1.1) almost everywhere. Hence,

$$
y(t)-y(0)=\int_{0}^{t} \eta(s) f(s, y(s), y(-s)) d s .
$$

Since the integrand is continuous on $(-T, T)$ it follows that the differential equation with reflection of the argument holds at each $t \in(-T, T)$ and so (1.1) has a solution $y \in C[-T, T] \cap C^{1}(-T, T)$.

To see the best possible nature of this result, consider the initial value problem

$$
\left\{\begin{array}{l}
y^{\prime}(t)=\eta(t) \hat{f}(t, y(t), y(-t)), \quad-T \leq t \leq T \\
y(0)=0
\end{array}\right.
$$

where $\hat{f}(t, y(t), y(-t))=(\psi(|y(t)|+|y(-t)|), 0, \cdots, 0)$. Suppose (1.4) has a solution $y=\left(y_{1}, \cdots, y_{n}\right)$ on the interval $[-T, T]$. Then $y_{i}(t) \equiv 0$ for $i=2, \cdots, n$ and hence $y_{1}^{\prime}(t)=\eta(t) \psi\left(\left|y_{1}(t)\right|+\left|y_{1}(-t)\right|\right)$ with $y_{1}(0)=0$. It follows that $y_{1}(t) \geq 0$ on $[0, T]$ and $y_{1}(t) \leq 0$ on $[-T, 0]$. In addition for $t \in[0, T]$ we have

$$
y_{1}^{\prime}(t)=\eta(t) \psi\left(y_{1}(t)-y_{1}(-t)\right)
$$

and

$$
y_{1}^{\prime}(-t)=\eta(-t) \psi\left(\left|y_{1}(-t)\right|+\left|y_{1}(t)\right|\right)=\eta(-t) \psi\left(-y_{1}(-t)+y_{1}(t)\right)
$$


Now (1.5) and (1.6) yield

$$
\frac{y_{1}^{\prime}(t)+y_{1}^{\prime}(-t)}{\psi\left(y_{1}(t)-y_{1}(-t)\right)}=\eta(t)+\eta(-t)
$$

and integration from 0 to $T$ gives

$$
\begin{aligned}
\int_{0}^{T}[\eta(t)+\eta(-t)] d t & =\int_{0}^{T} \frac{\left\{y_{1}^{\prime}(t)+y_{1}^{\prime}(-t)\right\}}{\psi\left(y_{1}(t)-y_{1}(-t)\right)} d t \\
& =\int_{0}^{y_{1}(T)-y_{1}(-T)} \frac{d u}{\psi(u)} \\
& =\int_{0}^{\left|y_{1}(T)\right|+\left|y_{1}(-T)\right|} \frac{d u}{\psi(u)}<\int_{0}^{\infty} \frac{d u}{\psi(u)} .
\end{aligned}
$$

EXAMPLE. (Linear and sublinear growth)

Consider

$$
\left\{\begin{array}{l}
y^{\prime}(t)=f(t, y(t), y(-t)), \quad-T \leq t \leq T \\
y(0)=r
\end{array}\right.
$$

where $f$ is continuous and $\left|f\left(t, u_{1}, u_{2}\right)\right| \leq A(t)\left(\left|u_{1}\right|+\left|u_{2}\right|\right)^{p}+B(t), p \leq 1$ for bounded functions $A(t), B(t) \geq 0$. If $A_{0}$ and $B_{0}$ are upper bounds for $A(t)$ and $B(t)$ respectively then,

$$
|f(t, y(t), y(-t))| \leq A_{0}(|y(t)|+|y(-t)|)^{p}+B_{0} .
$$

Let $\psi(u)=A_{0} u^{p}+B_{0}$ and since $\int_{2|r|}^{\infty} d u / \psi(u)=\infty,(1.7)$ has a solution on $[-T, T]$ for all $T>0$.

\section{Scalar Boundary Value Problems}

In this section we firstly consider second order differential equations with reflection of the argument, in just the function, with Sturm-Liouville boundary conditions at $t=-1$ and $t=1$, namely

$$
\left\{\begin{array}{l}
y^{\prime \prime}(t)=f\left(t, y(t), y(-t), y^{\prime}(t)\right), \quad-1 \leq t \leq 1 \\
-\alpha y(-1)+\beta y^{\prime}(-1)=r ; \quad \alpha>0, \beta \geq 0 \\
a y(1)+b y^{\prime}(1)=s ; \quad a>0, b \geq 0
\end{array}\right.
$$

where $f:[-1,1] \times R^{3} \rightarrow R$ is continuous. For simplicity we take $T=1$ in this section, corresponding results hold on any bounded interval $[-T, T]$. To establish existence results for (2.1), we introduce the related family of problems, for $0<\lambda<1$,

$$
\left\{\begin{array}{l}
y^{\prime \prime}(t)=\lambda f\left(t, y(t), y(-t), y^{\prime}(t)\right), \quad-1 \leq t \leq 1 \\
-\alpha y(-1)+\beta y^{\prime}(-1)=r \\
a y(1)+b y^{\prime}(1)=s .
\end{array}\right.
$$


In view of Theorem $0.6(\mathrm{C}),(2.1)$ will have a solution $y \in C_{\mathscr{B}}^{1}[-1,1]$, with $y^{\prime}$ absolutely continuous on $[-1,1]$ and such that the differential equation in $(2.1)$ holds for almost all $t$ in $[-1,1]$, where $\mathscr{B}$ signifies the boundary conditions $U_{1}(u)=$ $-\alpha u(-1)+\beta u^{\prime}(-1)=r, U_{2}(u)=a u(1)+b u^{\prime}(1)=s$ provided there is an a priori bound, independent of $\lambda$ in $(0,1)$, on solutions of $(2.1)_{\lambda}$ in the $C^{1}[-1,1]$ norm.

COROLLARY 2.1. In fact $y \in C_{\mathscr{B}}^{2}[-1,1]$.

PROOF. To see this note that if $y$ is a solution to (2.1) then $y^{\prime}$ is absolutely continuous on $[-1,1]$ and $y$ satisfies the differential equation in (2.1) almost everywhere. Hence

$$
y^{\prime}(t)-y^{\prime}(0)=\int_{0}^{t} f\left(s, y(s), y(-s), y^{\prime}(s)\right) d s .
$$

Since the integrand is continuous on $[-1,1]$ it follows that (2.1) holds at each $t \in$ $[-1,1]$ and consequently $y \in C^{2}[-1,1]$.

LEMMA 2.2. Let $f:[-1,1] \times R^{3} \rightarrow R$ be continuous and satisfy:

$$
\left\{\begin{array}{l}
\text { there is a constant } K \geq 0 \text { such that whenever } y \in C^{2}(-1,1) \text { and } \\
|y(s)| \text { achieves its maximum at } t \in(-1,1) \text { and }|y(t)|>K \text { then } \\
y(t) f(t, y(t), y(-t), 0)>0 .
\end{array}\right.
$$

Then each solution of $(2.1)_{\lambda}$ satisfies $|y(s)| \leq M_{0}=\max \{K,|s| / a,|r| / \alpha\}$ on $[-1,1]$.

PROOF. Let $y(s)$ be a solution of $(2.1)_{\lambda}$. If $|y(s)|$ achieves its maximum at a point $t_{0} \in(-1,1)$ then $y^{\prime}\left(t_{0}\right)=0$ and $y\left(t_{0}\right) y^{\prime \prime}\left(t_{0}\right) \leq 0$. Consequently

$$
0 \geq y\left(t_{0}\right) y^{\prime \prime}\left(t_{0}\right)=\lambda y\left(t_{0}\right) f\left(t_{0}, y\left(t_{0}\right), y\left(-t_{0}\right), 0\right)
$$

and by (2.2), $\left|y\left(t_{0}\right)\right| \leq K$. Now assume $|y(s)|$ takes its maximum at $t_{0}=1$. Then $y(1) y^{\prime}(1) \geq 0$ and so

$$
0 \geq-b y(1) y^{\prime}(1)=y(1)[a y(1)-s] \geq a|y(1)|^{2}-|s||y(1)| ;
$$

that is

$$
0 \geq|y(1)|\{a|y(1)|-|s|\}
$$

and so $|y(t)| \leq|y(1)| \leq|s| / a$ for $t \in[-1,1]$. Similarly if $|y(s)|$ takes its maximum at $t_{0}=-1$, then $|y(t)| \leq|y(-1)| \leq|r| / \alpha$ for $t \in[-1,1]$. Consequently, $|y(t)| \leq M_{0}$, $t \in[-1,1]$, as claimed.

We now place a Bernstein-Nagumo type growth restriction on $f$ to obtain a bound on $y^{\prime}$. Once this is done the existence of a solution to $(2.1)$ is guaranteed by the above ideas. Specifically we obtain the following theorem: 
THEOREM 2.3. Suppose $f:[-1,1] \times R^{3} \rightarrow R$ is continuous and satisfies (2.2). In addition, assume there is a function $\psi:[0, \infty) \rightarrow(0, \infty)$ such that $1 \backslash \psi$ is locally integrable on $[0, \infty)$,

$$
\left|f\left(t, u_{1}, u_{2}, p\right)\right| \leq \psi(|p|) \quad \text { for } \quad\left(t, u_{1}, u_{2}\right) \in[-1,1] \times\left[-M_{0}, M_{0}\right]^{2}
$$

and $\int_{c}^{\infty}(x / \psi(x)) d x>2 M_{0}$ where $M_{0}=\max \{K,|s| / a,|r| / \alpha\}$ and

$$
c= \begin{cases}\frac{|s|+a M_{0}}{b} & \text { if } b>0 \\ \frac{|r|+\alpha M_{0}}{\beta} & \text { if } b=0 \text { and } \beta>0 \\ \frac{1}{2}\left|\frac{s}{a}+\frac{r}{\alpha}\right| & \text { if } b=0 \text { and } \beta=0 .\end{cases}
$$

Then (2.1) has a solution $y \in C^{2}[-1,1]$.

PROOF. Once we show that there is a constant $M_{1}$ independent of $\lambda$ such that $\left|y^{\prime}(t)\right| \leq M_{1}$ on $[-1,1]$ for each solution $y(t)$ to $(2.1)_{\lambda}$, then Corollary 2.1, Lemma 2.2 and the above discussion guarantees a $C^{2}[-1,1]$ solution to $(2.1)$. Firstly, by Lemma 2.2, $|y(t)| \leq M_{0}$ for $t \in[-1,1]$. If $b>0$, then $\left|y^{\prime}(1)\right|=|s-a y(1)| / b \leq$ $\left(|s|+a M_{0}\right) / b$, while if $\beta>0,\left|y^{\prime}(-1)\right|=|r+\alpha y(-1)| / \beta \leq\left(|r|+\alpha M_{0}\right) / \beta$ and finally, if $b=\beta=0, y(1)=s / a$ and $y(-1)=-r / \alpha$, so there is a point $\tau \in(-1,1)$ at which $\left|y^{\prime}(\tau)\right|=\frac{1}{2}|s / a+r / \alpha|$. Thus, with $c$ defined as above, each $t \in[-1,1]$ for which $\left|y^{\prime}(t)\right|>c$ belongs to an interval $[\mu, \nu]$ such that $y^{\prime}$ maintains a fixed sign on $[\mu, \nu]$ and $\left|y^{\prime}(\mu)\right|$ and/or $\left|y^{\prime}(\nu)\right|$ equals $c$. To be definite, suppose $y^{\prime}>c$ on $(\mu, \nu)$ and $y^{\prime}(\mu)=c$.

Then the differential equation and the above growth condition yield

$$
\frac{y^{\prime}(x) y^{\prime \prime}(x)}{\psi\left(y^{\prime}(x)\right)} \leq y^{\prime}(x) \quad \text { for } \quad \mu \leq x \leq \nu .
$$

Integration from $\mu$ to $t$ yields

$$
\int_{c}^{y^{\prime}(t)} \frac{u}{\psi(u)} d u \leq y(t)-y(\mu) \leq 2 M_{0}<\int_{c}^{\infty} \frac{u}{\psi(u)} d u
$$

and we conclude that there is a constant $M_{1}^{*}$ independent of $\lambda \in(0,1)$ such that $y^{\prime}(t) \leq M_{1}^{*}$. The same bound is obtained by virtually identical reasoning if $y(\nu)=c$ and in the cases when $y^{\prime}<-c$. Thus we conclude that $\left|y^{\prime}(t)\right| \leq M_{1}=\max \left\{c, M_{1}^{*}\right\}$ on $[-1,1]$. 
EXAMPLE. The second order boundary value problem

$$
\left\{\begin{aligned}
y^{\prime \prime}(t)= & f\left(t, y(t), y(-t), y^{\prime}(t)\right)=a+b y(t)-c y(-t) \\
& +\left[y^{\prime}(t)\right]^{2} \sum_{i=1}^{n}\left\{d_{i} y^{p_{i}}(t)+l_{i} y^{q_{i}}(t) y^{r_{i}}(-t)+f_{i} y^{s_{i}}(-t)+g_{i}\right\} \\
& -1 \leq t \leq 1 \\
y(-1)= & y(1)=0
\end{aligned}\right.
$$

has a solution provided $b>c \geq 0, d_{i}, l_{i}, f_{i}, g_{i}, p_{i} \geq 0, q_{i} \geq 0, r_{i} \geq 0, s_{i} \geq 0$ and $a$ are constants.

To see this we apply Theorem 2.3. First $b=c+q$ for some $q>0$. Suppose $y \in C^{2}(-1,1)$ and the maximum of $|y(s)|$ occurs at some $t \in(-1,1)$.

Then

$$
y(t)\{y(t)-y(-t)\} \geq 0
$$

and thus

$$
\begin{aligned}
y(t) f(t, y(t), y(-t), 0) & =a y(t)+b y^{2}(t)-c y(-t) y(t) \\
& =a y(t)+c\{y(t)-y(-t)\} y(t)+q y^{2}(t) \\
& \geq a y(t)+q y^{2}(t)>0
\end{aligned}
$$

for $|y(t)|>|a| / q$. Hence $f$ satisfies condition (2.2). In addition it is clear that the growth rate restriction in Theorem 2.3 holds for $\psi(p)=A_{0}+B_{0} p^{2}$ for appropriate constants $A_{0}$ and $B_{0}$. Now since

$$
\int_{c}^{\infty} \frac{x}{\psi(x)} d x=\int_{0}^{\infty} \frac{x}{A_{0}+B_{0} x^{2}} d x=+\infty
$$

$\psi(p)$ satisfies all the conditions of Theorem 2.3 and so $(2.3)$ has a $C^{2}[-1,1]$ solution.

Small modifications in the proof of Theorem 2.3 permit the nonlinearity to be singular at $t=-1$ or $t=1$ or both. Consider

$$
\left\{\begin{array}{l}
y^{\prime \prime}(t)=\eta(t) f\left(t, y(t), y(-t), y^{\prime}(t)\right), \quad-1 \leq t \leq 1 \\
-\alpha y(-1)+\beta y^{\prime}(-1)=r ; \quad \alpha>0, \beta \geq 0 \\
a y(1)+b y^{\prime}(1)=s ; \quad a>0, b \geq 0
\end{array}\right.
$$

where $f:[-1,1] \times R^{3} \rightarrow R$ is continuous.

THEOREM 2.4. Let $1 / \eta:[-1,1] \rightarrow[0, \infty)$ be continuous with $\eta>0$ on $(-1,1)$ and $\eta$ integrable on $[-1,1]$. Suppose $f$ satisfies condition (2.2) and that there is a continuous function $\psi:[0, \infty) \rightarrow[0, \infty)$ with

$$
\left|f\left(t, u_{1}, u_{2}, p\right)\right| \leq \psi(|p|) \quad \text { for } \quad\left(t, u_{1}, u_{2}\right) \in[-1,1] \times\left[-M_{0}, M_{0}\right]^{2}
$$


and

$$
\int_{c}^{\infty} \frac{d u}{\psi(u)}>\int_{-1}^{1} \eta(s) d s
$$

where $M_{0}$ and $c$ are as in Theorem 2.3. Then (2.4) has a solution $y \in C^{1}[-1,1] \cap$ $C^{2}(-1,1)$.

PROOF. As usual, the proof reduces to establishing an a priori bound in $C^{1}[-1,1]$ on solutions of

$$
\left\{\begin{array}{l}
y^{\prime \prime}(t)=\lambda \eta(t) f\left(t, y(t), y(-t), y^{\prime}(t)\right), \quad-1 \leq t \leq 1 \\
-\alpha y(-1)+\beta y^{\prime}(-1)=r ; \quad \alpha>0, \beta \geq 0 \\
a y(1)+b y^{\prime}(1)=s ; \quad a>0, b \geq 0
\end{array}\right.
$$

where $0<\lambda<1$. Once this is done (2.4) has a solution $y$ and the reasoning of Corollary 2.1 applies to show that $y$ will have the added smoothness claimed above.

Now the bound $|y(t)| \leq M_{0}$ on $[-1,1]$ for solutions to $(2.4)_{\lambda}$ follows by the reasoning in Lemma 2.2 because $\eta>0$ on $(-1,1)$. Given this bound on $y$, just as in Theorem 2.3, each $t \in[-1,1]$ for which $\left|y^{\prime}(t)\right|>c$ determines an interval $\mu \leq s \leq t$ (or $t \leq s \leq \mu$ ) such that $\left|y^{\prime}(s)\right|>c$ except at $\mu$ and $\left|y^{\prime}(\mu)\right|=c$. To be definite assume the interval is $\mu \leq s \leq t$. Then

$$
\left|y^{\prime}(s)\right|^{\prime}=\frac{y^{\prime}(s) y^{\prime \prime}(s)}{\left|y^{\prime}(s)\right|} \leq\left|y^{\prime \prime}(s)\right| \quad \text { provided } \quad y^{\prime}(s) \neq 0
$$

and use of this estimate, yields

$$
\int_{c}^{\left|y^{\prime}(t)\right|} \frac{d u}{\psi(u)} \leq \int_{\mu}^{t} \frac{\left|y^{\prime \prime}(s)\right|}{\psi\left(\left|y^{\prime}(s)\right|\right)} d s \leq \int_{\mu}^{t} \eta(s) d s \leq \int_{-1}^{1} \eta(s) d s<\int_{c}^{\infty} \frac{d u}{\psi(u)} .
$$

Thus there is a constant $M_{1}^{*}$ such that $\left|y^{\prime}(t)\right| \leq M_{1}$ and $\left|y^{\prime}(t)\right| \leq \max \left\{c, M_{1}^{*}\right\} \equiv M_{1}$ on $[-1,1]$. Thus, solutions to $(2.4)_{\lambda}$ are bounded independent of $\lambda$ in $(0,1)$ in the $C^{1}[-1,1]$ norm and existence follows.

Finally, in this section we consider boundary value problems with reflection of the argument in both the function and its first derivative, namely

$$
\left\{\begin{array}{l}
y^{\prime \prime}(t)=f\left(t, y(t), y(-t), y^{\prime}(t), y^{\prime}(-t)\right), \quad-1 \leq t \leq 1 \\
-\alpha y(-1)+\beta y^{\prime}(-1)=r ; \quad \alpha>0 \text { and } \beta \geq 0 \\
a y(1)+b y^{\prime}(1)=s ; \quad a>0 \text { and } b \geq 0
\end{array}\right.
$$

where $f:[-1,1] \times R^{4} \rightarrow R$ is continuous. Once again we establish existence of a solution to $(2.5)$ by finding an a priori bound in $C^{1}[-1,1]$ on solutions to

$$
\left\{\begin{array}{l}
y^{\prime \prime}(t)=\lambda f\left(t, y(t), y(-t), y^{\prime}(t), y^{\prime}(-t)\right), \quad-1 \leq t \leq 1 \\
-\alpha y(-1)+\beta y^{\prime}(-1)=r \\
a y(1)+b y^{\prime}(1)=s
\end{array}\right.
$$

for $0<\lambda<1$. 
THEOREM 2.5. Let $f:[-1,1] \times R^{4} \rightarrow R$ be continuous. In addition assume:
There is a constant $K \geq 0$ such that wherever $y \in C^{2}(-1,1)$ and $|y(s)|$ achieves its maximum at $t \in(-1,1)$ and $|y(t)|>K$ then $y(t) f\left(t, y(t), y(-t), 0, y^{\prime}(-t)\right)>0$

There exists a constant $A \neq-1$ and continuous functions $g:[-1,1] \times$ $R^{2} \rightarrow R, h: R \rightarrow R$ and $k: R \rightarrow R$ such that

$$
\begin{aligned}
& f\left(t, y(t), y(-t), y^{\prime}(t), y^{\prime}(-t)\right) \\
& =A f\left(-t, y(-t), y(t), y^{\prime}(-t), y^{\prime}(t)\right)+g(t, y(t), y(-t)) \\
& \quad+h(y(t)) y^{\prime}(t)+k(y(-t)) y^{\prime}(-t)
\end{aligned}
$$

for all $t \in(0,1)$ and for any function $y \in C^{1}[-1,1]$. In addition let $L \geq 0$ be the smallest constant such that for $u, v, w \in\left[-M_{0}, M_{0}\right]$,

$$
\left|\int_{u}^{v} h(s) d s+\int_{w}^{u} k(s) d s\right| \leq L M_{0} .
$$

Here $M_{0}=\max \{K,|s| / a,|r| / \alpha\}$.

There is a continuous function $\psi:[0, \infty) \rightarrow(0, \infty)$ which is nondecreasing on $(0, \infty)$ and such that

$$
|f(t, u, v, p, w)| \leq \psi(|p|+|w|)
$$

for $(t, u, v) \in[-1,1] \times\left[-M_{0}, M_{0}\right]^{2}$ and also that

$$
2<\int_{2 N}^{\infty} \frac{d u}{\psi(u)}
$$

where

$$
N= \begin{cases}\frac{1}{|1+A|}\left\{Q+\left|\frac{s}{a}+\frac{A r}{\alpha}\right|\right\} & \text { if } \beta=b=0 \\ \frac{1}{|1+A|}\left\{Q+\frac{|r|}{\alpha}+|A| M_{0}\right\} & \text { if } \beta=0, b>0 \\ \frac{1}{|1+A|}\left\{Q+\frac{|s|}{a}+|A| M_{0}\right\} & \text { if } \beta>0, b=0 \\ \frac{1}{|1+A|}\{R+X+|A| Y\} & \text { if } \beta>0, b>0 .\end{cases}
$$

Here $R=L M_{0}+M^{*} / 2, Q=R+|A-1| M_{0}, X=\left(|s|+a M_{0}\right) / b$, $Y=\left(|r|+\alpha M_{0}\right) / \beta$ and $M^{*}=\sup |g(t, u, v)|$ with the supremum computed over $[-1,1] \times\left[-M_{0}, M_{0}\right]^{2}$. 
Then (2.5) has a solution $y \in C^{2}[-1,1]$.

PROOF. For any solution $y$ to (2.5) $)_{\lambda}$ assumption (2.6) implies that $|y(t)| \leq M_{0}$, $t \in[-1,1]$ by essentially the same reasoning used in Lemma 2.2 . We turn next to put a bound on $y^{\prime}$ and the idea is to first bound $y^{\prime}(0)$. Now (2.7) implies

$$
y^{\prime \prime}(t)=A y^{\prime \prime}(-t)+\lambda g(t, y(t), y(-t))+\lambda h(y(t)) y^{\prime}(t)+\lambda k(y(-t)) y^{\prime}(-t)
$$

for $t \in(0,1)$ and with $M^{*}$ as defined above,

$$
\left|y^{\prime \prime}(t)-A y^{\prime \prime}(-t)-\lambda h(y(t)) y^{\prime}(t)-\lambda k y(-t) y^{\prime}(-t)\right| \leq M^{*} .
$$

Integration from 0 to $t$, with the fact that $\left|\int_{0}^{1} j(s) d s\right| \leq \int_{0}^{1}|j(s)| d s$ for any integrable function $j$ on $[0,1]$, yields,

$$
\left|\left[y^{\prime}(t)+A y^{\prime}(-t)-(1+A) y^{\prime}(0)\right]-\lambda\left\{\int_{y(0)}^{y(t)} h(s) d s+\int_{y(-t)}^{y(0)} k(s) d s\right\}\right| \leq M^{*} t .
$$

Also since ||$a|-| b|| \leq|a-b|$ we have

$$
\left|y^{\prime}(t)+A y^{\prime}(-t)-(1+A) y^{\prime}(0)\right| \leq L M_{0}+M^{*} t .
$$

Integrate finally from 0 to 1 to obtain

(2.10) $\left|y^{\prime}(0)\right| \leq \frac{1}{|1+A|}\left\{L M_{0}+\frac{M^{*}}{2}+|y(1)-A y(-1)+(A-1) y(0)|\right\}$.

If $\beta=b=0, \beta=0$ and $b>0$ or $\beta>0$ and $b=0$, then clearly (2.10) implies $\left|y^{\prime}(0)\right| \leq N$. On the other hand if $\beta>0$ and $b>0$, (2.9) with $t=1$ implies

$$
\left|y^{\prime}(0)\right| \leq \frac{1}{|1+A|}\left\{L M_{0}+M^{*}+\left|y^{\prime}(1)+A y^{\prime}(-1)\right|\right\} \leq N
$$

since $y^{\prime}(1)=(s-a y(1)) / b$ and $y^{\prime}(-1)=(r+\alpha y(-1)) / \beta$. Consequently in all cases

$$
\left|y^{\prime}(0)\right| \leq N .
$$

Now following the ideas of Theorem 1.1 we see immediately that for $t \in(0,1)$

$$
\left|y^{\prime}(t)\right|+\left|y^{\prime}(-t)\right| \leq 2 N+\int_{-t}^{t}\left|y^{\prime \prime}(s)\right| d s
$$

and consequently

$$
\frac{\left|y^{\prime \prime}(t)\right|+\left|y^{\prime \prime}(-t)\right|}{\psi\left(2 N+\int_{-t}^{t}\left|y^{\prime \prime}(s)\right| d s\right)} \leq 2
$$


Integration from 0 to 1 gives with $\rho(t)=2 N+\int_{-t}^{t}\left|y^{\prime \prime}(s)\right| d s$,

$$
\int_{2 N}^{\rho(t)} \frac{d u}{\psi(u)} \leq 2<\int_{2 N}^{\infty} \frac{d u}{\psi(u)}
$$

and we obtain a bound on $y^{\prime}$ independent of $\lambda \in(0,1)$ just as in Theorem 1.1.

REMARK. It is also possible to replace the equation that $f$ has to satisfy in (2.7) by

$$
\begin{array}{rl}
f\left(t, y(t), y(-t), y^{\prime}(t), y^{\prime}(-t)\right) \\
=A & f\left(-t, y(-t), y(t), y^{\prime}(-t), y^{\prime}(t)\right)+g(t, y(t), y(-t)) \\
+ & \sum_{i=1}^{n} h_{i}(y(t))\left\{y^{\prime}(t)\right\}^{\alpha_{i}}+\sum_{i=1}^{m} k_{i}(y(-t))\left\{y^{\prime}(-t)\right\}^{\beta_{i}}
\end{array}
$$

where $0 \leq \alpha_{i}, \beta_{i} \leq 1$ and obtain existence of a solution to (2.5) with appropriate adjustments in the assumption. However, we choose to omit the details; the trick is to use Hölders integral inequality if an $\alpha_{i}$ or $\beta_{i}$ is less than 1.

REMARK. We can use similar techniques to obtain an analogue of Theorem 2.5 for problems of the form

$$
\left\{\begin{array}{l}
y^{\prime \prime}(t)=\eta(t) f\left(t, y(t), y(-t), y^{\prime}(t), y^{\prime}(-t)\right) \quad-1 \leq t \leq 1 \\
-\alpha y(-1)+\beta y^{\prime}(-1)=r ; \quad \alpha>0 \text { and } \beta \geq 0 \\
a y(1)+b y^{\prime}(1)=s ; \quad a>0 \text { and } b \geq 0
\end{array}\right.
$$

where $f:[-1,1] \times R^{4} \rightarrow R, \frac{1}{\eta}:[-1,1] \rightarrow[0, \infty)$ are continuous with $\eta>0$ on $(-1,1)$ and $\eta$ is integrable on $[-1,1]$. However, since the ideas and strategy are more or less the same (the only major difference is that we replace $h(y(t)) y^{\prime}(t)$ and $k(y(-t)) y^{\prime}(-t)$ in $(2.7)$ by $h(y(t))\left[y^{\prime}(t)\right]^{1 / p}$ and $k(y(-t))\left[y^{\prime}(-t)\right]^{1 / p}$ where $p>1$ and assume $\eta \in L^{q}[-1,1]$ with $q=p /(p-1)$ and $\eta(t)=\eta(-t)$ for $t \in(0,1)$; of course the trick in the proof is now to use Hölder's integral inequality) we choose to omit the details.

\section{Boundary Value Problems for Systems}

Consider systems of second order differential equations with reflection of the argument in both the function and its first derivative and with Dirichlet boundary data,

$$
\left\{\begin{array}{l}
y^{\prime \prime}(t)=f\left(t, y(t), y(-t), y^{\prime}(t), y^{\prime}(-t)\right), \quad-1 \leq t \leq 1 \\
y(-1)=0 \\
y(1)=0
\end{array}\right.
$$


and the related family of problems

$$
\left\{\begin{array}{l}
y^{\prime \prime}(t)=\lambda f\left(t, y(t), y(-t), y^{\prime}(t), y^{\prime}(-t)\right), \quad-1 \leq t \leq 1 \\
y(-1)=0 \\
y(1)=0
\end{array}\right.
$$

where $f:[-1,1] \times R^{4 n} \rightarrow R^{n}$ is an $L^{p}$-Carathéodory function, $0<\lambda<1$. For $x, y \in R^{n}$ let $x . y$ denote the usual inner product. First however recall Wirtinger's inequalities:

\section{LEMMA 3.1.}

(i) If $u(x) \in C^{1}[a, b]$ and $u(a)=u(b)=0$, then $\|u\|^{2} \leq(b-a)^{2} / \pi^{2}\left\|u^{\prime}\right\|$.

(ii) If $u(x) \in C^{1}[a, b]$ and $u(a)=0$, then $\|u\|^{2} \leq 4(b-a)^{2} / \pi^{2}\left\|u^{\prime}\right\|^{2}$.

THEOREM 3.2. Assume $f(t, u, v, p, w)=g(t, u, v, p, w)+h(t, u, v, p, w)$ with

$$
u \cdot g(t, u, v, p, w) \geq A|u|^{2}+B u \cdot p+C|u||v|+D|u||p|+E|u||w|
$$

for all $(t, u, v, p, w) \in[-1,1] \times R^{4 n}$ and

$$
|g(t, u, v, p, w)| \leq F(t, u, v)|p|^{2}+G(t, u, v)|w|^{2}+H(t, u, v)
$$

where $F, G$ and $H$ are bounded on bounded sets

and

$$
\left\{\begin{array}{l}
|h(t, u, v, p, w)| \leq M\left(|u|^{\alpha}+|v|^{\beta}+|p|^{\gamma}+|w|^{\tau}\right) \text { for constants } M, \alpha \\
\beta, \gamma \text { and } \tau \text { with } 0 \leq \alpha, \beta \gamma, \tau<1
\end{array}\right.
$$

Then (3.1) has a solution $y \in W^{2, p}[-1,1]$ in each of the following cases:

(a) $A \geq 0, \quad 4|C|+2 \pi(|D|+|E|)<\pi^{2}$

(b) $A<0, \quad 4|C|+2 \pi(|D|+|E|)<\pi^{2}+4 A$.

PROOF. As usual, we need only establish a priori bounds in $C^{1}[-1,1]$ on solutions $y(t)$ to $(3.1)_{\lambda}$. Now integration-by-parts and use of the boundary data yields

$$
\int_{-1}^{1} y(t) \cdot y^{\prime \prime}(t) d t=-\int_{-1}^{1}\left|y^{\prime}(t)\right|^{2} d t \quad \text { and } \quad \int_{-1}^{1} y(t) \cdot y^{\prime}(t) d t=0 .
$$

Thus taking the dot product of the differential equation in $(3.1)_{\lambda}$ with $y(t)$ and using (3.2) leads to 


$$
\begin{aligned}
\int_{-1}^{1}\left|y^{\prime}(t)\right|^{2} d t \leq- & A \lambda \int_{-1}^{1}|y(t)|^{2} d t+|C| \int_{-1}^{1}|y(t)||y(-t)| d t \\
& +|D| \int_{-1}^{1}|y(t)|\left|y^{\prime}(t)\right| d t+|E| \int_{-1}^{1}|y(t)|\left|y^{\prime}(-t)\right| d t \\
& +\int_{-1}^{1}|y(t)|\left|h\left(t, y(t), y(-t), y^{\prime}(t), y^{\prime}(-t)\right)\right| d t
\end{aligned}
$$

Now using Cauchy's inequality, $a b \leq \frac{\epsilon}{2} a^{2}+\frac{1}{2 \epsilon} b^{2}$, we find

$$
\begin{aligned}
& \int_{-1}^{1}|y(t)|\left|h\left(t, y(t), y(-t), y^{\prime}(t), y^{\prime}(-t)\right)\right| d t \\
& \quad \leq \frac{\epsilon}{2} \int_{-1}^{1}|y(t)|^{2} d t+\frac{1}{2 \epsilon} \int_{-1}^{1}\left|h\left(t, y(t), y(-t), y^{\prime}(t), y^{\prime}(-t)\right)\right|^{2} d t .
\end{aligned}
$$

Also since $(a+b+c+d)^{2} \leq 8\left(a^{2}+b^{2}+c^{2}+d^{2}\right)$, (3.3) yields

$$
\begin{aligned}
& \left|h\left(t, y(t), y(-t), y^{\prime}(t), y^{\prime}(-t)\right)\right|^{2} \\
& \quad \leq 8 M^{2}\left\{|y(t)|^{2 \alpha}+|y(-t)|^{2 \beta}+\left|y^{\prime}(t)\right|^{2 \gamma}+\left|y^{\prime}(-t)\right|^{2 \tau}\right\},
\end{aligned}
$$

and Hölder inequality gives

$$
\int_{-1}^{1}|y(t)|^{2 \delta} d t \leq 2\left(\int_{-1}^{1}|y(t)|^{2} d t\right)^{\delta}=2\|y(t)\|^{2 \delta} \equiv 2\|y\|^{2 \delta}
$$

for any $\delta$ in $[0,1]$. In addition the change-of-variables formula yields

$$
\int_{-1}^{1}|y(-t)|^{2 \delta} d t=\int_{-1}^{1}|y(t)|^{2 \delta} d t \quad \text { and } \quad \int_{-1}^{1}\left|y^{\prime}(-t)\right|^{2 \delta} d t=\int_{-1}^{1}\left|y^{\prime}(t)\right|^{2 \delta} d t
$$

for any $\delta$ in $[0,1]$. Use of these estimates gives

$$
\begin{aligned}
\int_{-1}^{1}\left|y(t) \| h\left(t, y(t), y(-t), y^{\prime}(t), y^{\prime}(-t)\right)\right| d t \\
\leq \frac{\epsilon}{2}\|y\|^{2}+\frac{8 M^{2}}{\epsilon}\left\{\|y\|^{2 \alpha}+\|y\|^{2 \beta}+\left\|y^{\prime}\right\|^{2 \gamma}+\left\|y^{\prime}\right\|^{2 \tau}\right\} .
\end{aligned}
$$

In addition Hölder's integral inequality and (3.5) gives

$$
\begin{aligned}
& |C| \int_{-1}^{1}\left|y(t)\left\|y(-t)|d t \leq| C\left|\|y\|\left(\int_{-1}^{1}|y(-t)|^{2} d t\right)^{\frac{1}{2}}=\right| C \mid\right\| y \|^{2},\right. \\
& |D| \int_{-1}^{1}\left|y(t)\left\|y^{\prime}(t)|d t \leq| D \mid\right\| y\|\| y^{\prime} \| \quad\right. \text { and } \\
& |E| \int_{-1}^{1}\left|y(t)\left\|y^{\prime}(-t)|d t \leq| E \mid\right\| y\|\| y^{\prime} \| .\right.
\end{aligned}
$$


Substitute (3.6) and (3.7) into (3.5) to obtain

$$
\begin{aligned}
\left\|y^{\prime}\right\|^{2} \leq- & A \lambda\|y\|^{2}+|C|\|y\|^{2}+|D|\|y\|\left\|y^{\prime}\right\|+|E|\|y\|\left\|y^{\prime}\right\| \\
& +\frac{\epsilon}{2}\|y\|^{2}+\frac{8 M^{2}}{\epsilon}\left\{\|y\|^{2 \alpha}+\|y\|^{2 \beta}+\left\|y^{\prime}\right\|^{2 \gamma}+\left\|y^{\prime}\right\|^{2 \tau}\right\} .
\end{aligned}
$$

Next apply Wirtinger's inequality, $\|y\|^{2} \leq \frac{4}{\pi^{2}}\left\|y^{\prime}\right\|^{2}$, to get

$$
\begin{aligned}
& \left(1-\frac{4|C|}{\pi^{2}}-\frac{2(|D|+|E|)}{\pi}-\frac{2 \epsilon}{\pi^{2}}\right)\left\|y^{\prime}\right\|^{2} \\
& \leq-A \lambda\|y\|^{2}+\frac{8 M^{2}}{\epsilon}\left\{\left(\frac{2}{\pi}\right)^{2 \alpha}\left\|y^{\prime}\right\|^{2 \alpha}+\left(\frac{2}{\pi}\right)^{2 \beta}\left\|y^{\prime}\right\|^{2 \beta}+\left\|y^{\prime}\right\|^{2 \gamma}+\left\|y^{\prime}\right\|^{2 \tau}\right\} .
\end{aligned}
$$

Now consider Case (a) with $A \geq 0$ and $4|C|+2 \pi(|D|+|E|)<\pi^{2}$ that is $1-4|C| / \pi^{2}-2(|D|+|E|) / \pi>0$. With these assumptions we can delete the $A \lambda$ term from (3.8) and then fix $\epsilon>0$ suitably small so that the term is brackets on the left hand side of (3.8) is positive. This done, (3.8) and $\alpha, \beta, \gamma, \tau<1$ implies there exists a constant $M_{1}$ independent of $\lambda$ such that

$$
\left\|y^{\prime}\right\| \leq M_{1} \text {. }
$$

Now consider Case (b) with $A<0$ and $4|C|+2 \pi(|D|+|E|)<\pi^{2}+4 A$ that is $1-4|C| / \pi^{2}-2(|D|+|E|) / \pi+4 A / \pi^{2}>0$. Since $A<0$ we have $-A \lambda \leq-A$ and applying Wirtinger's inequality yields

$$
-A \lambda\|y\|^{2} \leq-A\|y\|^{2} \leq-\frac{4 A}{\pi^{2}}\left\|y^{\prime}\right\|^{2} .
$$

Substitute this estimate in (3.8) to obtain

$$
\begin{aligned}
(1 & \left.-\frac{4|C|}{\pi^{2}}-\frac{2(|D|+|E|)}{\pi}+\frac{4 A}{\pi^{2}}-\frac{2 \epsilon}{\pi^{2}}\right)\left\|y^{\prime}\right\|^{2} \\
& \leq \frac{8 M^{2}}{\epsilon}\left\{\left(\frac{2}{\pi}\right)^{2 \alpha}\left\|y^{\prime}\right\|^{2 \alpha}+\left(\frac{2}{\pi}\right)^{\beta}\left\|y^{\prime}\right\|^{2 \beta}+\left\|y^{\prime}\right\|^{2 \gamma}+\left\|y^{\prime}\right\|^{2 \tau}\right\} .
\end{aligned}
$$

Now fix $\epsilon>0$ suitably small so that the term in brackets on the left of (3.10) is positive. As above this leads to (3.9) for a certain constant $M_{1}$ independent of $\lambda$ in $(0,1)$.

From (3.10) and $y(-1)=0$, we find that

$$
|y(t)|=\left|\int_{-1}^{t} y^{\prime}(s) d s\right| \leq 2\left\{\int_{-1}^{1}\left|y^{\prime}(s)\right|^{2} d s\right\}^{\frac{1}{2}} \leq 2 M_{1} \equiv M_{0}
$$


for all $t \in[-1,1]$, which is the required a priori bound on $y(t)$. Given this bound and (3.2), we can determine constants $F, G$ and $H$ (dependent only on $M_{1}$ ) such that $|g(t, u, v, p, w)| \leq F|p|^{2}+G|w|^{2}+H \quad$ for all $(t, u, v) \in[-1,1] \times\left[-M_{0}, M_{0}\right]^{2}$.

This estimate, the boundedness of $y$, and the differential equation yields $\left|y^{\prime \prime}(t)\right| \leq$ $I\left|y^{\prime}(t)\right|^{2}+J\left|y^{\prime}(-t)\right|^{2}+K$ for almost all $t$ in $[-1,1]$ and constants $I, J$ and $K$ (depending only on $M, \alpha, \beta, \gamma, \tau$ and $M_{0}$ ). Integration from -1 to 1 gives $\int_{-1}^{1}\left|y^{\prime \prime}(t)\right| d t \leq I \int_{-1}^{1}\left|y^{\prime}(t)\right|^{2} d t+J \int_{-1}^{1}\left|y^{\prime}(-t)\right|^{2} d t+2 K \leq(I+J) M_{1}^{2}+2 K \equiv M_{2}$. Let $y=\left(y_{1}, \cdots, y_{n}\right)$. Since $\int_{-1}^{1}\left|y_{i}^{\prime \prime}(t)\right| d t \leq \int_{-1}^{1}\left|y^{\prime \prime}(t)\right| d t$ and $y_{i}^{\prime}\left(c_{i}\right)=0$ for some $c_{i} \in(0,1)$, we infer that

$$
\left|y_{i}^{\prime}(t)\right|=\left|\int_{c_{i}}^{t} y_{i}^{\prime \prime}(s) d s\right| \leq M_{2} \quad \text { for } t \in[-1,1] .
$$

and so $\left|y^{\prime}(t)\right| \leq \sqrt{n} M_{2}$ for $t \in[-1,1]$. Thus, all required $a$ priori bounds are obtained and a solution to (3.1) exists.

We next examine systems of second order equations with reflection of the argument with a Sturm-Liouville boundary condition at $t=1$ and a Dirichlet condition at $t=-1$.

REMARK. Similar results can be obtained if we have a Sturm-Liouville condition at $t=-1$ and a Dirichlet condition at $t=1$.

Now consider the Sturm-Liouville problem

$$
\left\{\begin{array}{l}
y^{\prime \prime}(t)=f\left(t, y(t), y(-t), y^{\prime}(t), y^{\prime}(-t)\right), \quad-1 \leq t \leq 1 \\
y(-1)=0 \\
a y(1)+b y^{\prime}(1)=0 ; \quad a>0, b>0
\end{array}\right.
$$

and the related family of problems

$$
(3.11)_{\lambda} \quad \begin{cases}y^{\prime \prime}(t)=\lambda f\left(t, y(t), y(-t), y^{\prime}(t), y^{\prime}(-t)\right), & -1 \leq t \leq 1 \\ y(-1)=0 & \\ a y(1)+b y^{\prime}(1)=0 & \end{cases}
$$

where $f:[-1,1] \times R^{4 n} \rightarrow R^{n}$ is an $L^{p}$-Carathéodory function and $0<\lambda<1$.

THEOREM 3.3. Assume $f$ satisfies (3.2), with $B=0$, and (3.3). Then (3.11) has a solution $y \in W^{2, p}[0,1]$ in each of the following cases:

(a) $A \geq 0, \quad 16|C|+4 \pi(|D|+|E|)<\pi^{2}$

(b) $A<0, \quad 16|C|+4 \pi(|D|+|E|)<\pi^{2}+16 A$. 
PROOF. Integration by parts yields for any solution $y(t)$ to $(3.11)_{\lambda}$,

$$
-\int_{-1}^{1} y(t) \cdot y^{\prime \prime}(t) d t=-y(1) \cdot y^{\prime}(1)+\left\|y^{\prime}\right\|^{2}=\frac{a}{b}|y(1)|^{2}+\left\|y^{\prime}\right\|^{2} \geq\left\|y^{\prime}\right\|^{2}
$$

and (3.5) holds as before. In addition as in the proof of Theorem 3.2 we notice that (3.5), (3.6) and (3.7) hold, so we have

$$
\begin{gathered}
\left\|y^{\prime}\right\|^{2} \leq-A \lambda\|y\|^{2}+|C|\|y\|^{2}+\{|D|+|E|\}\|y\|\left\|y^{\prime}\right\|+\frac{\epsilon}{2}\|y\|^{2} \\
+\frac{8 M^{2}}{\epsilon}\left\{\|y\|^{2 \alpha}+\|y\|^{2 \beta}+\left\|y^{\prime}\right\|^{2 \gamma}+\left\|y^{\prime}\right\|^{2 \tau}\right\} .
\end{gathered}
$$

Now apply Lemma 3.1 (ii) to get

$$
\begin{aligned}
& \left(1-\frac{16|C|}{\pi^{2}}-\frac{4(|D|+|E|)}{\pi}-\frac{8 \epsilon}{\pi^{2}}\right)\left\|y^{\prime}\right\|^{2} \\
& \leq-A \lambda\|y\|^{2}+\frac{8 M^{2}}{\epsilon}\left\{\left(\frac{4}{\pi}\right)^{2 \alpha}\left\|y^{\prime}\right\|^{2 \alpha}+\left(\frac{4}{\pi}\right)^{2 \beta}\left\|y^{\prime}\right\|^{2 \beta}+\left\|y^{\prime}\right\|^{2 \gamma}+\left\|y^{\prime}\right\|^{2 \tau}\right\}
\end{aligned}
$$

and the result follows by essentially the same reasoning as in Theorem 3.2.

The only change in the argument occurs at the very end. Obtain $M_{0}, M_{1}$ and $M_{2}$ as in Theorem 3.2 and let $y=\left(y_{1}, \cdots, y_{n}\right)$. Now since $a y_{i}(1)+b y_{i}^{\prime}(1)=0$ we have $\left|y_{i}^{\prime}(1)\right| \leq a M_{0} / b$ and consequently

$$
\left|y_{i}^{\prime}(t)\right| \leq \frac{a M_{0}}{b}+\int_{t}^{1}\left|y_{i}^{\prime \prime}(s)\right| d s \leq \frac{a M_{0}}{b}+M_{2} \quad \text { for } t \in[-1,1]
$$

and so $\left|y^{\prime}(t)\right| \leq \sqrt{n}\left\{a M_{0} / b+M_{2}\right\}$ for $t \in[-1,1]$.

REMARK. A similar result can be obtained for the boundary value problem (3.11) if $a=0$ (that is $y^{\prime}(1)=0$ ).

REMARK. It is possible to combine the ideas in Theorems 3.2, 3.3, together with the ideas in [15] to obtain theorems similar to those deduced in [15] for problems of the form (3.1), (3.11) when $f$ is singular at $t=0$ or $t=1$ or both.

REMARK. One can improve the results in Theorem 3.3 if the Raleigh Ritz minimization theorem is used instead of Lemma 3.1(ii). 


\section{Systems of boundary Value Problems of Bernstein-Carathéodory Type}

In this section we obtain analogues of the results in Section 2 for systems of boundary value problems where our nonlinear term is a Carathéodory function. In particular, consider

$$
\left\{\begin{array}{l}
y^{\prime \prime}(t)=f\left(t, y(t), y(-t), y^{\prime}(t)\right), \quad-1 \leq t \leq 1 \\
-\alpha y(-1)+\beta y^{\prime}(-1)=r ; \quad \alpha>0, \beta \geq 0 \\
\text { ay(1)+by }(1)=s ; \quad a>0, b \geq 0
\end{array}\right.
$$

where $f:[-1,1] \times R^{3 n} \rightarrow R^{n}$ is an $L^{p}$-Carathéodory function. As usual the existence of a solution to (4.1) follows once a priori bounds in $C^{1}[-1,1]$ are established for the related family

$$
\left\{\begin{array}{l}
y^{\prime \prime}(t)=\lambda f\left(t, y(t), y(-t), y^{\prime}(t)\right), \quad-1 \leq t \leq 1 \\
-\alpha y(-1)+\beta y^{\prime}(-1)=r \\
a y(1)+b y^{\prime}(1)=s
\end{array}\right.
$$

for $0<\lambda<1$.

\section{THEOREM 4.1. Suppose}

(a) There is a constant $M$ such that $|u|>M$ implies $u \cdot f(t, u, v, p)>0$

(b) The equations in (4.1) can be listed so that for the $j^{\text {th }}$ equation, there are functions $A_{j}\left(t, u, v, p_{1}, \cdots, p_{j-1}\right)$ and $B_{j}\left(t, u, v, p_{1}, \cdots, p_{j-1}\right)$ which are bounded on bounded sets of $[-1,1] \times R^{2 n} \times R^{j-1}$ and satisfy

$$
\left|f_{j}(t, u, v, p)\right| \leq A_{j}\left(t, u, v, p_{1}, \cdots, p_{j-1}\right) p_{j}^{2}+B_{j}\left(t, u, v, p_{1}, \cdots, p_{j-1}\right)
$$

where $f=\left(f_{1}, \cdots, f_{n}\right)$ and $p=\left(p_{1}, \cdots, p_{n}\right)$ and for $j=1, A_{1}$ and $B_{1}$ do not depend on the $p$ variables.

Then (4.1) has a solution in $W^{2, p}[-1,1]$.

PROOF. Let $y(t)$ be a solution to (4.1) $)_{\lambda}$ and suppose $r(t)=\frac{1}{2}|y(t)|^{2}$ has a maximum on $[-1,1]$ which is greater than $M_{0}=\max \left\{M^{2},|s|^{2} / a^{2},|r|^{2} / \alpha^{2}\right\}$. Now if the maximum of $r(t)$ occurs at 1 , then $y(1) \cdot y^{\prime}(1)=r^{\prime}(1) \geq 0$ and so $a y(1) \cdot y(1)+b y^{\prime}(1)$. $y(1)=s \cdot y(1)$ implies $|y(1)| \leq|s| / a$. Similarly, if the maximum of $r(t)$ occurs at -1 , then $|y(-1)| \leq|r| / a$. Hence, by choice of $M_{0}, r(t)$ achieves its maximum at $c$ in $(-1,1)$ at which $r^{\prime}(c)=0$. Now (a) implies

$$
r^{\prime \prime}(t)=\lambda y(t) \cdot f\left(t, y(t), y(-t), y^{\prime}(t)\right)+\left|y^{\prime}(t)\right|^{2}>0
$$


for almost all $t$ in $[c, c+d]$ where $d>0$ is fixed so that $|y(t)|>M$ on $[c, c+d]$. Thus for $t \in[c, c+d]$,

$$
r^{\prime}(t)=\int_{c}^{t} r^{\prime \prime}(s) d s>0 \quad \text { and } \quad r(t)-r(c)=\int_{c}^{t} r^{\prime}(s) d s>0,
$$

which contradicts the maximality. Thus $|y(t)| \leq M_{0}$ on $[-1,1]$. It remains to bound $y^{\prime}$ on $[-1,1]$. By (b) with $j=1$ there are constants $a_{1}$ and $b_{1}$ such that $\left|y_{1}^{\prime \prime}\right| \leq a_{1}\left(y_{1}^{\prime}\right)^{2}+b_{1}$ almost everywhere on $[-1,1]$, here $y=\left(y_{1}, \cdots, y_{n}\right)$. Moreover, since $y_{1}$ is bounded by $M_{0}$, the mean value theorem implies there exists $\tau_{1} \in(-1,1)$ such that $\left|y_{1}^{\prime}\left(\tau_{1}\right)\right| \leq M_{0}$. Now following the ideas in Theorem 2.3 we see that there exists a constant $M_{1}^{\prime}$ independent of $\lambda$ such that $\left|y_{1}^{\prime}(t)\right| \leq M_{1}^{\prime}$ on [-1, 1]. Arguing inductively, we deduce bounds of $M_{i}^{\prime}$ on $y_{i}^{\prime}$ for each $i=1, \cdots, n$ and hence obtain a bound $M^{\prime}$ such that $\left|y^{\prime}(t)\right| \leq M^{\prime}$ for $t$ in $[-1,1]$.

REMARK. In assumption (a), $u \cdot f>0$ can be replaced by $u \cdot f \geq 0$. To see this let $\delta=\sup \{d \in[c, 1]:|y(t)|>M$ on $[c, c+d]\}$. Then $|y(c+\delta)|=M$. From (4.2) on $\left[c, c+\delta\right.$ ) we have $r^{\prime \prime}(t) \geq\left|y^{\prime}(t)\right|^{2}$ and we get a contradiction, as before, unless $y^{\prime}(t)=0$ almost everywhere on $[c, c+\delta)$; however, in the latter case $y(c+\delta)=y(c)$, so $|y(c+\delta)|>M$, a contradiction.

Combining the ideas of Corollary 2.1, Lemma 2.2 and Theorem 4.1, we immediately obtain:

THEOREM 4.2. Let $f:[-1,1] \times R^{3 n} \rightarrow R^{n}$ be a continuous function and assume:

(a) There is a constant $M$ such that $|u|>M$ and $u \cdot p=0$ implies

$$
u \cdot f(t, u, v, p)>0 \text {. }
$$

In addition, suppose (b) of Theorem 4.1 is satisfied. Then (4.1) has a solution $y \in C^{2}[-1,1]$.

Next, we consider a system in which $f$ may be singular at $t=-1$ or $t=1$ or both. Consider

$$
\left\{\begin{array}{l}
y^{\prime \prime}(t)=\eta(t) f\left(t, y(t), y(-t), y^{\prime}(t)\right), \quad-1 \leq t \leq 1 \\
-\alpha y(-1)+\beta y^{\prime}(-1)=0 ; \quad \alpha>0, \beta \geq 0 \\
a y(1)+b y^{\prime}(1)=0 ; \quad a>0, b \geq 0
\end{array}\right.
$$

where $f:[-1,1] \times R^{3 n} \rightarrow R^{n}, \frac{1}{\eta}:[-1,1] \rightarrow[0, \infty)$ are continuous with $\eta>0$ on $(-1,1)$ and $\eta$ integrable on $[-1,1]$. 
THEOREM 4.3. Let $f$ and $\eta$ be as above and suppose (a) of Theorem 4.2 is satisfied. In addition, suppose $f(t, u, v, p)$ is bounded for $(t, u, v)$ varying in a bounded set. Then (4.2) has a solution $y$ in $C^{1}[-1,1] \cap C^{2}(-1,1)$.

ProOF. The proof reduces to establishing a priori bounds in the $C^{1}[-1,1]$ norm on solutions of

$$
\left\{\begin{array}{l}
y^{\prime \prime}(t)=\lambda \eta(t) f\left(t, y(t), y(-t), y^{\prime}(t)\right), \quad-1 \leq t \leq 1 \\
-\alpha y(-1)+\beta y^{\prime}(-1)=0 \\
a y(1)+b y^{\prime}(1)=0
\end{array}\right.
$$

where $0<\lambda<1$ and, of course, the added smoothness follows from the ideas of Corollary 2.1. Combining the ideas of Lemma 2.2 and Theorem 4.1 with the fact that $\eta>0$ on $(-1,1)$ immediately yields $|y(t)| \leq M_{0}=\max \left\{M^{2},|s|^{2} / a^{2},|r|^{2} / \alpha^{2}\right\}$ on $[-1,1]$ for solutions $y=\left(y_{1}, \cdots, y_{n}\right)$ to $(4.2)_{\lambda}$. In addition, there exist $M^{*}$ independent of $\lambda$ such that $\left|f\left(t, y(t), y(-t), y^{\prime}(t)\right)\right| \leq M^{*}$. Then $\left|y^{\prime \prime}(t)\right| \leq \eta(t) M^{*}$ for $t \in(-1,1)$. Since $\left|y_{i}^{\prime \prime}(t)\right| \leq\left|y^{\prime \prime}(t)\right|$ and $\left|y_{i}^{\prime}\left(\tau_{i}\right)\right| \leq M_{0}$ for some $\tau_{i} \in(-1,1)$ we find for each $i$,

$$
\left|y_{i}^{\prime}(t)\right| \leq M_{0}+\left|\int_{c_{i}}^{t} \eta(s) d s\right| M^{*} \leq M_{0}+M^{*} \int_{-1}^{1} \eta(s) d s=M^{* *} .
$$

Consequently, $\left|y^{\prime}(t)\right| \leq \sqrt{n} M^{* *}, t \in[-1,1]$.

In the case of scalar equations we can sharpen the result in Theorem 4.1.

THEOREM 4.4. Let $f:[-1,1] \times R^{3} \rightarrow R$ be an $L^{p}$-Carathéodory function and assume

(a) There exist, $M>0$ such that $|u| \geq M$ implies

$$
u f(t, u, v, 0)>0 \quad \text { a.e. on }[-1,1] \text {. }
$$

(b) There is a Borel function $\psi:[0, \infty) \rightarrow(0, \infty)$ such that $|f(t, u, v, p)| \leq \psi(|p|)$ for a.e. $t$ in $[-1,1]$ and all $u, v \in\left[-M_{0}, M_{0}\right]$ and such that $\int_{c}^{\infty}(x / \psi(x)) d x>$ $2 M_{0}$ where $M_{0}=\max \{M,|s| / a,|r| / \alpha\}$ and

$$
c=\left\{\begin{array}{lll}
\frac{|s|+a M_{0}}{b} & \text { if } & b>0 \\
\frac{|r|+\alpha M_{0}}{\beta} & \text { if } & b=0 \text { and } \beta>0 \\
\frac{1}{2}\left|\frac{s}{a}+\frac{r}{\alpha}\right| & \text { if } & b=0 \text { and } \beta=0
\end{array}\right.
$$


Then (4.1) has a solution in $W^{2, p}[-1,1]$.

ProOF. Since the proof of this theorem involves only minor alterations in the corrsponding proof in [7] or [9, 10] for regular second order boundary value problems, we choose as a result to omit the details.

\section{References}

[1] A. R. Aftabizadeh, Y. K. Huang and J. Wiener, 'Bounded solutions for differential equations with reflection of the argument', J. Math. Anal. Appl. 135 (1988), 31-37.

[2] A. R. Aftabizadeh and J. Wiener, 'Boundary value problems for differential equations with reflection of argument', Internat. J. Math. Math. Sci. 8 (1985), 151-163.

[3] L. E. Bobisud, D. O'Regan and W. D. Royalty, 'Singular boundary value problems', Applicable Anal. 23 (1986), 233-243.

[4] J. Dugundji and A. Granas, Fixed point theory, Vol. 1, Monografhie Matematyczne (PWN, Warsaw, 1982).

[5] M. Frigon, A. Granas and Z. Guennoun, 'Sur l'intervalle maximal d'existence de solutions pour des inclusions différentialles', C. R. Acad. Sci. Paris Sér. I Math. 306 (1988), 747-750.

[6] M. Frigon and D. O'Regan, 'On a generalization of a theorem of S. Bernstein', Ann. Polon. Math. 48 (1988), 297-306.

[7] A. Granas and Z. Guennoun, 'Quelques résultats dans la thérie de Bernstein-Carathéodory de l'equations $y^{\prime \prime}=f\left(t, y, y^{\prime}\right)$ ', C.R. Acad. Sci. Paris Sér. I Math. 306 (1988), 703-706.

[8] A. Granas, R. B. Guenther and J. W. Lee, 'Nonlinear boundary value problems for ordinary differential equations', Dissertationes Mathematicae (Warsaw, 1985).

[9] —_ 'Existence for classical and Carathéodory solutions of nonlinear systems and applications', in: Proceedings of the international conference on theory and applications of differential equations (Ohio University Press, Athens, 1988) pp. 353-364.

[10] - 'Some general existence principles in the Caratheodory theory of nonlinear differential systems', J. Math. Pures Appl. 70 (1991), 153-196.

[11] C. P. Gupta, 'Boundary value problems for differential equations in Hilbert spaces involving reflection of the argument', J. Math. Anal. Appl. 128 (1987), 375-388.

[12] - 'Existence and uniqueness theorems for boundary value problems involving reflection of the argument', Nonlinear Anal. 11 (1987), 1075-1083.

[13] J. W. Lee and D. O'Regan, 'Topological Transversality: Applications to initial value problems', Ann. Polon. Math. 48 (1988), 247-252.

[14] - 'Existence results for differential delay equations I', J. Differential Equations 102 (1993), 342-359.

[15] D. O'Regan, 'Second and higher order systems of boundary value problems', J. Math. Anal. Appl. 156 (1991), 120-149.

[16] D. Przeworska-Rolewicz, 'Equations with transformed argument - an algebraic approach' (Panstwowe Wydawnictwo Naukowe, Warsaw, 1973).

\section{Department of Mathematics}

University College Galway

Galway

Ireland 